\title{
Fases de la cadena de suministro de las empresas avícolas
}

\author{
Aponte F., Bertila J.* \\ González, Abraham J.** \\ González P., Angel D.***
}

\section{Resumen}

En la cadena de suministro de las empresas avícolas se realiza una gran cantidad de procesos que originan una brecha de tiempo y espacio entre el insumo y el producto final, dificultando el adecuado análisis de sus elementos. Por esto, el objetivo del presente artículo es analizar las fases de la misma, específicamente en lo correspondiente a la producción de pollo. La investigación fue de tipo analítica, no experimental; con un diseño transeccional. La muestra estuvo conformada por 4 empresas avícolas del estado Zulia, siendo los gerentes informantes clave en la investigación. Los resultados indican que la fase de aprovisionamiento de la cadena de suministro avícola posee 9 eslabones que aplican alianzas comerciales, tecnológicas y de producción para mantenerse integrados. La fase de procesamiento está compuesta por 2 eslabones que operan bajo una estrategia de producción continua. Finalmente, la fase de distribución posee 4 eslabones, que conforman canales de distribución de varios intermediarios que distribuyen los productos de forma intensiva. Se concluye que la industria avícola posee una cadena de suministro compleja, formada por 15 eslabones que se encargan de producir, procesar y distribuir diferentes productos a los consumidores en el momento, cantidad y condiciones deseadas.

Palabras clave: Cadena de suministro, empresa avícola, aprovisionamiento, procesamiento, distribución.

\section{Recibido: 01-11-12. Aceptado: 14-03-13}

* Licenciada en Contaduría Pública (Universidad del Zulia -LUZ, Venezuela). Magíster en Gerencia de Mantenimiento (LUZ, Venezuela). Profesor titular del Departamento de Producción, Escuela de Industrial, Facultad de Ingeniería (LUZ), e-mail: berty_ve@yahoo.com, bertyaponte@cantv.net

** Ingeniero Industrial. Doctor en Ciencias Gerenciales (URBE, Venezuela). Profesor agregado del Departamento de Producción, Escuela de Industrial, Facultad de Ingeniería (LUZ), e-mail: ajgonzalez060276@yahoo.es

*** Ingeniero industrial. Facultad de Ingeniería (LUZ), e-mail: angeldavidgp2009@hotmail.com 


\title{
Phases in the Supply Chain for Poultry Enterprises
}

\begin{abstract}
In the supply chain for poultry companies, a large number of processes are carried out that cause a time and space gap between raw material input and the final product, making an adequate analysis of its elements difficult. The objective of this article is to analyze these phases, specifically as related to producing chicken. Research was of the analytic type, with a non-experimental, cross-sectional design. The sample consisted of four (4) poultry companies in the State of Zulia; their managers were key research informants. Results indicate that the provisioning phase in the poultry supply chain has nine links that apply commercial, technological and production alliances to keep them integrated. The processing phase is composed of two links that operate under a continuous production strategy. Finally, the distribution phase has four links that constitute distribution channels of various intermediaries, which distribute products in an intensive manner. Conclusions are that the poultry industry has a complex supply chain consisting of fifteen links that take charge of producing, processing and distributing different products to consumers at the moment and in the quantity and conditions desired.
\end{abstract}

Keywords: Supply chain, poultry company, provisioning, processing, distribution.

\section{Introducción}

En la actualidad, el ámbito empresarial ha sido afectado por constantes cambios generados, entre otros, por: los avances tecnológicos, la globalización de los negocios y las modificaciones en el ámbito político, económico y social; alterando los criterios tradicionales de gestión organizacional. Todo esto ha conllevado a un ambiente competitivo que a cualquier empresa se le dificulta la producción de los bienes o servicios a ofrecer si trabaja de manera aislada; por tanto, es necesaria la actuación en conjunto de diferentes organizaciones a lo largo del proceso para hacer llegar el producto al consumidor final de la manera más económica y eficiente posible.

Ahora bien, con la finalidad de generar ventajas competitivas, todo tipo de empresa grande, mediana o pequeña de cualquier sector, requiere continuamente incrementar su nivel de repuestas ante los cambios que presenta la demanda en el nuevo contexto de la economía global. Es imprescindible que éstas conozcan cuáles son las tendencias y prioridades sobre las que tendrán que trabajar para garantizar su permanencia en el medio en el cual se encuentran; no obstante, el adecuado desempeño de una organización y de su sistema logístico, ya no depende de sí misma, sino también de sus proveedores, distribuidores, clientes y todos aquellos que forman parte de su cadena de suministro.

La cadena de suministro es definifa por Miquel et al. (2008:435), como "la unión de todas las empresas que participan en la producción, distribución, manipulación, almacenamiento y comercialización de un producto; es decir, integra todas las empresas que hacen posible 
que un producto salga al mercado". BaIlou (2004), añade que se refiere a la relación e integración de todas las actividades asociadas a los productos, desde la adquisición de materia prima hasta la entrega del mismo al consumidor final, para crear una ventaja competitiva sólida.

En este contexto, la industria avícola nacional se caracteriza por realizar un número considerable de procesos, entre los cuales se incluyen la fecundación, cría y el beneficio de las aves, e incluso la elaboración del alimento balanceado para las misma; por lo que, desde el momento en que se inicia hasta que culmina el proceso de producción, existe una gran cantidad de tiempo y de actividades que incrementan el grado de complejidad para gestionar su cadena de suministro.

La carne de pollo es de suma importancia para Venezuela y el mundo dado que forma parte de un producto de la cesta básica. Según el Instituto Nacional de Estadísticas (2011), existe un incremento sostenido en el consumo nacional. En los últimos años ha aumentado en un 3\%; es decir, el consumo incrementó de 5.946.632 toneladas en el segundo semestre de 2009 a 6.110 .627 toneladas en el segundo semestre de 2010.

Es de destacar que, una inadecuada gestión de la cadena de suministro ocasiona, entre otros, retrasos en los trámites para obtener divisas necesarias para la compra de productos importados; fluctuaciones en la distribución del producto; malas relaciones con proveedores y comercializadores; altos costos de producción; incluso paradas por falta de materia prima; así como retrasos en los tiempos de entrega del producto final al cliente; afectando de forma directa la rentabilidad de la em- presa y la competitividad en el mercado al ofrecer productos de baja calidad y/o de elevado costo. Todo esto conlleva a posibles sanciones emanadas por el Ejecutivo Nacional producto de la Ley de Costos y Precios Justos, incurriendo en multas o cierre temporal de la empresa.

Debido a la gran importancia y necesidad que la carne de pollo representa para el mercado nacional y por el hecho de ser parte importante de la alimentación de la sociedad, es necesario analizar las fases que componen la cadena de suministro de la industria avícola. Con el propósito de lograr este objetivo, la investigación se cataloga como analítica, definida por Hurtado (2007:103), como "aquella que trata de entender las situaciones en términos de las relaciones de sus componentes". Este tipo de investigación permite analizar los elementos que conforman el tema en estudio y la manera en la que están conectados.

De igual forma, el diseño de la investigación es no experimental, la cual según Hernández et al. (2006:25) comprende "un estudio que se realiza sin la manipulación deliberada de variables y en los que solo se observan los fenómenos en su ambiente natural para después analizarlos".

Con respecto a la temporalidad es transeccional contemporáneo, porque se realiza en un único momento del tiempo. Hernández et al. (2006:208) explican que "los diseños de investigación transeccional o transversal recopilan datos en un sólo momento, en un tiempo único. Su propósito es describir variables y analizar su incidencia e interrelación en un momento dado". A su vez, se considera con un diseño de campo, debido a que todos los datos e información necesarios para 
la investigación fueron tomados de fuentes directas en su contexto natural durante el año 2012.

La población está conformada por todas las empresas avícolas que operan en el estado Zulia. Al respecto Chirinos et al, (2008) indican que en el estado Zulia operan 6 empresas avícolas que poseen integradas gran parte de su cadena de suministro: Avícola de Occidente (AVIDOCA), Avícola La Rosita (AVIROSA), Protinal del Zulia, Agropecuaria Nivar (AGRONIVAR), Procesadora Industrial de Pollo (PINPOLLO) y Productores Avícolas del Zulia (PROAVE). En este estudio se toma una muestra por conveniencia compuesta por las siguientes empresas: AVIDOCA, AVIROSA, Protinal y AGRONIVAR. Las empresas mencionadas fueron las que estuvieron dispuestas a participar en la investigación. Para la recolección de datos se emplea un cuestionario con 21 preguntas, el cual es aplicado a gerentes de las 4 empresas mencionadas.

Durante el estudio se analizan las fases de aprovisionamiento, procesamiento y distribución de la cadena de suministro. En cuanto al aprovisionamiento, se estudia el canal de abastecimiento de la empresa, las alianzas que la misma posee con sus proveedores y las estrategias empleadas para llevar a cabo esta fase. Desde el punto de vista de procesamiento, se estudia cómo está compuesta y las estrategias que se aplican para la obtención del producto final. Finalmente, en la fase de distribución se estudian los canales que son empleados para hacer llegar el producto a los consumidores, así como la intensidad de distribución del producto.

\section{Generalidades de la cadena de suministro avícola}

En las organizaciones y los mercados, la cadena de suministro es considerada como un elemento clave de competitividad, debido a la importancia que tiene para lograr los resultados deseados en la misma, entre los que se pueden mencionar el margen de beneficio, los plazos de entrega, la calidad del servicio, la satisfacción del cliente, entre otros aspectos, aunado a la colaboración entre las empresas que persiguen un posicionamiento estratégico común y pretenden mejorar su eficiencia operativa (Bowersox et al., 2007).

Por suparte, Christopher (2008:93) establece que la cadena de suministro "está formada por el conjunto de empresas que están vinculadas, a través de relaciones con otras empresas, en los diferentes procesos y actividades que generan valor en forma de productos y servicios para el consumidor final".

Mientras que Chopra y Meindl (2008:3) dan una definición de cadena de suministro más amplia y detallada, considerando que existe una mayor cantidad de partes implicadas en la misma, al explicar que "una cadena de suministro está formada por todas aquellas partes involucradas de manera directa o indirecta en la satisfacción de una solicitud de un cliente".

Asimismo, Bowersox et al. (2007:5) la definen como la "colaboración de varias firmas que considera un sistema de flujos y restricciones de recursos importantes... Por lo tanto, se integran las operaciones de negocios desde la adquisición inicial de materiales hasta la entrega de productos y servicios a los clientes". Además agrega que mediante esta com- 
binación de esfuerzos, es posible cumplir con los compromisos hechos con los clientes de la organización, así como mejores relaciones con las redes de proveedores y de distribución, lo cual significa una ventaja competitiva para la empresa.

Considerando los elementos aportados por los diversos autores, se puede decir que la cadena de suministro es el conjunto de empresas o eslabones interrelacionados que realizan actividades a través de las cuales fluye el producto, la información y otros insumos necesarios. Dichas actividades están involucradas desde el momento de proveer la materia prima, hasta la entrega al consumidor final del producto terminado. Esta tiene como objetivo añadirle valor al producto a medida que pasa por cada uno de sus eslabones, buscando satisfacer plenamente las necesidades del cliente.

Definida la cadena de suministro, se hace evidente la gran importancia que ésta representa, respecto a lo cual Serra (2005:152) afirma: "lo que crea valor para el consumidor final no es la empresa que se encuentra en el último eslabón de la cadena, sino la combinación de las actuaciones de todos los miembros que la componen".

En tal sentido, según Ballou (2004: 5) la administración sistemática y estratégica de la cadena de suministro conlleva a "mejorar el desempeño a largo plazo de las empresas individuales y de la cadena de suministros como un todo". Mientras que Chopra y Meindl (2008:6) manifiestan que "una eficaz administración de la cadena de suministro comprende la administración de los activos y de los flujos de productos, información y fondos de la cadena para maximizar la rentabilidad total de la misma".

Por ende, es de gran importancia tener pleno conocimiento de cada una de las partes que integran la cadena de suministro, para así determinar las funciones que cumplen al igual que las diversas características que la identifican; de tal manera que se puedan establecer límites y centrar esfuerzos en la mejora continua, logrando la optimización de los procesos y con ello la satisfacción de los clientes.

El hacer un seguimiento a todo el proceso, así como recabar, analizar y utilizar la información necesaria durante el mismo, permite obtener procesos adecuados y eficientes a través de todo el flujo del producto, que garantizan un mayor rendimiento de los recursos, lo cual implica una mayor rentabilidad de la empresa así como su perduración en el tiempo con niveles de competencia adecuados.

En el caso de la industria avícola, su cadena de suministro está compuesta por un gran número de procesos que incluyen el levante, reproducción, incubación, engorde, procesamiento, distribución y comercialización de todos sus productos. Algunas organizaciones poseen integrados todos los procesos mencionados; mientras que otras sólo realizan algunos de ellos.

Las organizaciones que realizan dichos procesos son las que forman parte de la cadena de suministro de la industria, los cuales se muestran en el Diagrama 1; ellos pertenecen a alguna de sus fases, según los objetivos que cada una persigue. Pires y Carretero (2007) mencionan que en general la cadena de suministro está compuesta por 3 fases: 


\section{Diagrama 1 \\ Eslabones de la cadena de suministro avícola}

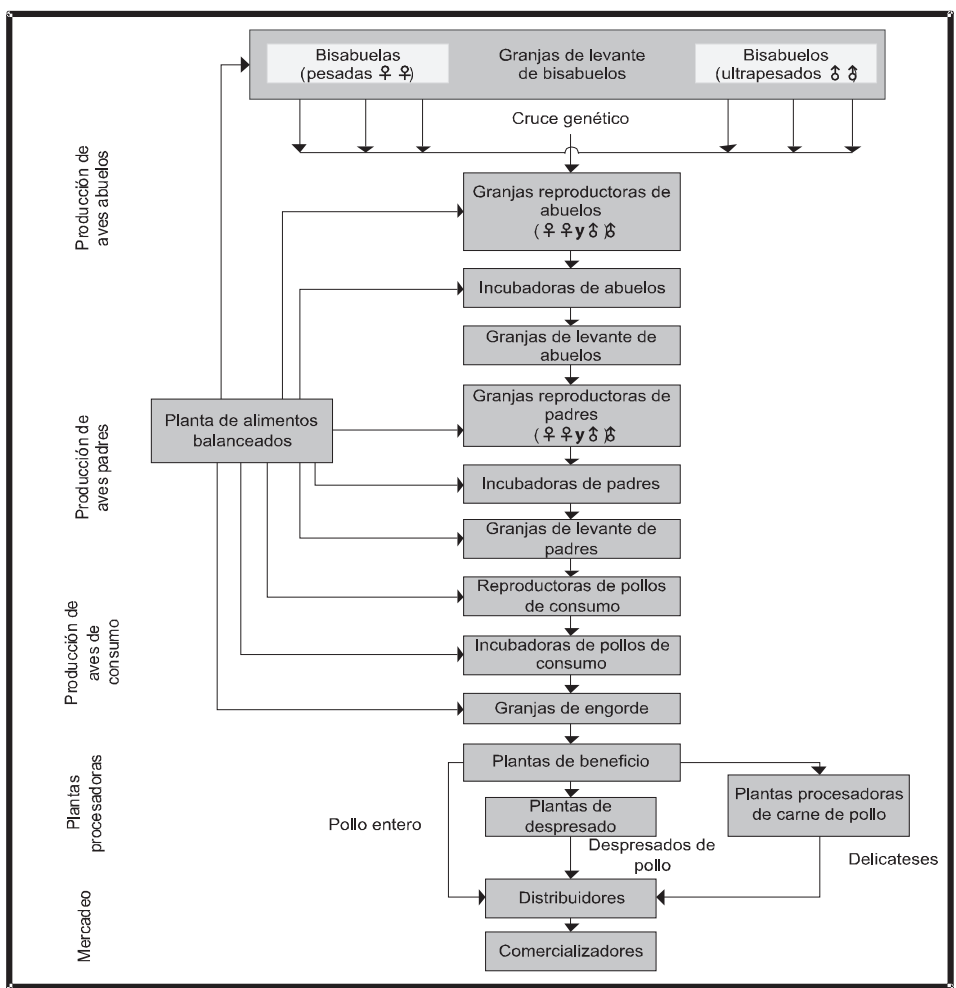

Fuente: Adaptado de Chirinos et al. (2008).

- Fase de aprovisionamiento: comprende los lugares donde se obtienen las materias primas; gestionando las mismas entre los puntos de adquisición (proveedores iniciales) y las plantas de procesamiento.

- Fase de procesamiento: donde los materiales son transformados para convertirlos en producto terminado.

- Fase de distribución: mediante la cual se traslada el producto final hasta los lugares de venta para que sea almacenado y posteriormente adquirido por el consumidor.

\section{Fase de aprovisionamiento de la cadena de suministro avícola}

Esta fase incluye todas las empresas que forman parte del canal de abastecimiento de la cadena de suministro avícola, el cual según Pires y Carretero (2007:25) "es el encargado de surtir de materiales a aquellas empresas que tendrán la tarea de procesarlos". En dicho canal se da el proceso de abastecimiento de la empresa; es decir, en él se realizan las actividades que intentan asegurar la 
obtención de materiales de alta caldiad y bajo costo que fluirán en la cadena para posteriormente ser transformados en un producto de valor para el cliente.

La investigación realizada indicó que la cadena de suministro de las empresas del sector avícola posee un canal de abastecimiento de varios niveles, el cual según Pires y Carretero (2007) es el que comprende varios proveedores (niveles) por los que deben pasar la materia prima hasta estar lista para ser utilizada o procesada. A continuación se describen los niveles o eslabones que forman parte del canal de abastecimiento:

- Proveedores de noveno nivel: integrado por los proveedores que se encargan de suministrar a la planta de alimento balanceado todos los ingredientes necesarios para la elaboración de sus productos: macroingredientes, polvos de harina de diferentes tipos, químicos líquidos, entre otros. De igual forma también comprende los proveedores de vacunas para las diferentes aves.

- Proveedores de octavo nivel: integrado por las granjas progenitoras y la planta de alimento balanceado.
En las granjas progenitoras se realizan los cruces de diferentes razas de gallos y gallinas, las cuales se manipulan genéticamente para obtener aves con las características deseadas: engorde rápido y de buena postura. En estas granjas se realiza la producción de las aves que son las abuelas de los pollos de consumo. Las mismas representan uno de los proveedores externos de la cadena de suministro avícola, porque a las empresas les parece más rentable adquirir las aves en este nivel que contratar personal especializado en el área de genética para poder realizar el cruce de razas.

La planta de alimentos balanceados es la encargada de elaborar los diferentes tipos de alimentos para aves de diferentes edades; es decir, este eslabón es el encargado de proveer alimentos a todas las aves que pasan por las granjas de la empresa. El Cuadro 1, muestra la información de las plantas de alimentos de las empresas estudiadas.

- Proveedores de séptimo nivel: integrado por las granjas de levante de abuelas que se encargan de la cría de las aves abuelas durante 24 sema-

\section{Plantas de alimentos balanceados}

\begin{tabular}{|c|c|c|c|}
\hline Nombre & $\begin{array}{c}\text { Capacidad } \\
\text { de producción }\end{array}$ & Tipo de alimento & Mercado \\
\hline Planta ABA (AVIDOCA) & 12.000 ton/mes & Alimento para aves & Interno \\
\hline $\begin{array}{l}\text { Planta de alimentos balanceados } \\
\text { Avícola La Rosita }\end{array}$ & 9.000 ton/mes & Alimento para aves & Interno \\
\hline Planta de alimentos AGRONIVAR & 8.000 ton $/ \mathrm{mes}$ & Alimento para aves & Interno \\
\hline $\begin{array}{l}\text { Planta de alimentos balanceados } \\
\text { Protinal del Zulia }\end{array}$ & 12.000 ton/mes & $\begin{array}{c}\text { Alimento para aves, } \\
\text { vacunos, cerdos } \\
\text { y conejos }\end{array}$ & $\begin{array}{l}\text { Interno } \\
\text { y externo }\end{array}$ \\
\hline
\end{tabular}

Fuente: Elaboración propia. 
nas. En este tiempo las aves se alimentan siguiendo un programa especial que varía según su edad; de igual forma se le colocan los medicamentos necesarios para preservar su correcto crecimiento. Entre las empresas estudiadas la única que tiene integrado este eslabón es Protinal del Zulia; cuenta con la granja de levante Proavica que posee una capacidad de 120.000 aves para el levante de abuelas; esta granja pertenece al complejo avícola Proavica.

- Proveedores de sexto nivel: conformado por las granjas de reproducción de padres en donde se colocan las aves abuelas cuando llegan a 24 semanas de edad y se mantienen 40 semanas, período durante el cual se da la producción de los huevos fértiles que contienen los futuros padres de las aves de consumo. Entre las empresas estudiadas sólo Protinal del Zulia posee integrado este nivel; su granja reproductora Proavica posee una capacidad de 120.000 aves para la producción de huevos fértiles; esta granja pertenece al complejo avícola Proavica.

- Proveedores de quinto nivel: integrado por las incubadoras de padres donde los huevos son colocados durante 19 días en las máquinas incubadoras para que reciban el calor necesario, y luego, son llevados a las máquinas nacedoras en la cual nacen los pollitos en un período de 2 días. Los pollitos son clasificados según su género y trasladados al siguiente nivel. Al igual que en los casos anteriores, solo Protinal del Zulia posee integrado este nivel; su incubadora Proavica puede incubar 3.289.466 huevos. Para el resto de las empresas este nivel es un proveedor externo que surte de pollitos a las granjas de levante.

- Proveedores de cuarto nivel: constituido por las granjas de levante de padres, las cuales se encargan de la cría durante 24 semanas de las aves que serán padres de las aves de consumo. Operan bajo las mismas características de las granjas de levante ya mencionadas. La Tabla 1, muestra las granjas de levante de padres de las empresas estudiadas,

Tabla 1

Granjas de levante de padres

\begin{tabular}{llcc}
\hline Empresa & Nombre & Cantidad de galpones & Capacidad (aves/24sem) \\
\hline AVIDOCA & Milagrosa 1 & 6 & 30.000 \\
& Milagrosa 2 & 6 & 30.000 \\
& Vista Alegre & 6 & 30.000 \\
& Coromoto & 5 & 28.000 \\
AVIROSA & Doña Alicia & 3 & 40.000 \\
& Zuliande & 12 & 101.000 \\
AGRONIVAR & Tucuabo & 3 & 6.500 \\
PROTINAL & Moruy & 4 & 120.000 \\
& Balzamal & 4 & 120.000 \\
\hline
\end{tabular}

Fuente: Elaboración Propia. 
la cantidad de galpones y la cantidad de aves que puede albergar.

- Proveedores de tercer nivel: integrado por las granjas de reproducción de pollos de consumo, en ellas se colocan las aves padres cuando llegan a 24 semanas de edad y se mantienen 40 semanas, período durante el cual se da la producción de los huevos que contienen las aves de consumo. La Tabla 2, muestra la información de las granjas de reproducción de pollos de consumo de las empresas estudiadas.

- Proveedores de segundo nivel: conformado por las incubadoras de los pollos de consumo; este nivel realiza el mismo trabajo que las incubadoras ya mencionadas, solo que aquí se manejan los huevos que contienen los pollos que serán vendidos para el consumo. La Tabla 3, muestra la información de las incubadoras de pollos de consumo de las empresas estudiadas.
Tabla 3

Incubadoras de pollos de consumo

\begin{tabular}{llr}
\hline Empresa & Nombre & $\begin{array}{c}\text { Capacidad } \\
\text { (huevos/ } \\
\text { 19días) }\end{array}$ \\
\hline AVIDOCA & Incubadora & 1.800 .000 \\
AVIROSA & Mara & 928.800 \\
& La Bonita & 952.560 \\
AGRONIVAR & Fertinivar & 74.250 \\
PROTINAL & Abudure & 2.933 .760 \\
& Atamaica & 2.933 .760 \\
\hline
\end{tabular}

Fuente: Elaboración Propia.

- Proveedores de primer nivel: constituido por las granjas de engorde en las que se reciben los pollitos recién nacidos procedentes de las incubadoras de pollos de consumo. Se encargan de engordar las aves durante 42 días empleando para ello un riguroso programa de alimentación que varía la

Tabla 2

\section{Granjas de reproducción de pollos de consumo}

\begin{tabular}{llcc}
\hline Empresa & Nombre & Cantidad de galpones & Capacidad (aves/40sem) \\
\hline AVIDOCA & C. Bolívar 1 & 4 & 32.000 \\
& C. Bolívar 2 & 4 & 32.000 \\
& Caracoles & 7 & 31.500 \\
& Mesa Grande & 5 & 31.500 \\
& Quebrada & 4 & 23.000 \\
& Tural 1 & 5 & 30.000 \\
AVIROSA & Tural 2 & 5 & 30.000 \\
& Doña Alicia & 6 & 66.000 \\
AGRONIVAR & La Bonita & 32 & 176.000 \\
PROTINAL & Tucuabo & 5 & 5.500 \\
& Abudure & 4 & 120.000 \\
& Atamaica & 4 & 120.000 \\
\hline
\end{tabular}

Fuente: Elaboración propia. 
composición de los alimentos según la edad del ave. Transcurrido este tiempo las aves se encuentran listas para ser procesadas; es decir, es el eslabón encargado de entregar la materia prima de la industria avícola a las plantas procesadoras, por ello es el último de la fase de aprovisionamiento. La Tabla 4, muestra la información de las granjas de engorde propias de las empresas estudiadas.

Este nivel también incluye a las diversas empresas que se encargan de surtir a la fase de procesamiento de materiales para el empaque y embalado de los productos.

El Cuadro 2, sintetiza los eslabones de la fase de aprovisionamiento que posee integrado cada empresa. Como

Tabla 4

Granjas de engorde

\begin{tabular}{|c|c|c|c|}
\hline Empresa & Nombre & Cantidad de galpones & Capacidad (aves/42días) \\
\hline \multirow[t]{2}{*}{ AVIDOCA } & Ciénaga & 60 & 600.000 \\
\hline & Galápago & 30 & 840.000 \\
\hline \multirow[t]{11}{*}{ AVIROSA } & Santa Ana & 6 & 222.000 \\
\hline & $3 \mathrm{M}$ & 6 & 222.000 \\
\hline & San José & 6 & 63.360 \\
\hline & José Gregorio & 3 & 24.000 \\
\hline & La Azul & 8 & 64.000 \\
\hline & Altagracia & 9 & 57.600 \\
\hline & San Martín & 10 & 85.440 \\
\hline & Las cruces & 4 & 23.040 \\
\hline & Buenos Aires & 3 & 24.000 \\
\hline & Obregón & 9 & 60.000 \\
\hline & Zenit & 4 & 44.000 \\
\hline \multirow[t]{6}{*}{ AGRONIVAR } & Jobo Alto & 16 & 112.000 \\
\hline & La Mancorna & 8 & 24.000 \\
\hline & Los Manolos & 4 & 24.000 \\
\hline & Los Romanes & 5 & 14.000 \\
\hline & Los Migueles & 4 & 24.000 \\
\hline & Ceciliana & 4 & 8.000 \\
\hline \multirow[t]{5}{*}{ PROTINAL } & Cocuiza & 50 & 950.000 \\
\hline & Santa Elena & 12 & 300.000 \\
\hline & Jagüey de León & 17 & 221.000 \\
\hline & Apolo & 13 & 235.000 \\
\hline & Curarire & 7 & 65.000 \\
\hline
\end{tabular}

Fuente: Elaboración propia. 


\section{Cuadro 2}

Eslabones de la fase de aprovisionamiento

\begin{tabular}{lcccc}
\hline \multicolumn{1}{c}{ Eslabones } & AVIDOCA & AVIROSA & PROTINAL & AGRONIVAR \\
\hline Planta de alimentos balanceados & $\mathrm{X}$ & $\mathrm{X}$ & $\mathrm{X}$ & $\mathrm{X}$ \\
Granjas de levante de abuelas & & & $\mathrm{X}$ & \\
Granjas reproductoras de padres & & & $\mathrm{X}$ & \\
Incubadora de padres & & & $\mathrm{X}$ & \\
Granjas de levante de padres & $\mathrm{X}$ & $\mathrm{X}$ & $\mathrm{X}$ & $\mathrm{X}$ \\
Granjas reproductoras de aves de consumo & $\mathrm{X}$ & $\mathrm{X}$ & $\mathrm{X}$ & $\mathrm{X}$ \\
Incubadoras de aves de consumo & $\mathrm{X}$ & $\mathrm{X}$ & $\mathrm{X}$ & $\mathrm{X}$ \\
Granjas de engorde & $\mathrm{X}$ & $\mathrm{X}$ & $\mathrm{X}$ & $\mathrm{X}$ \\
\hline
\end{tabular}

Fuente: Elaboración propia.

fue mencionado, las granjas de progenitores representan a los proveedores externos de la cadena de suministro avícola. De hecho, la investigación revela que de las empresas objeto de estudio sólo Protinal del Zulia posee en su fase de aprovisionamiento el complejo que se encarga de la producción de los padres (granjas de abuelas) adquiriendo los pollitos para las granjas de levante de proveedores de Brasil; el resto de las empresas adquieren los pollitos para el levante (aves padres) de proveedores externos nacionales o internacionales.

Los eslabones mostrados en el cuadro anterior se mantienen integrados por medio de alianzas estratégicas. La alianza consiste en un compromiso de trabajo conjunto de las organizaciones, en búsqueda de la mejora continua, mediante acciones encaminadas en la misma dirección para lograr metas comunes, propiciadas por una comunicación asertiva, con el objetivo de desarrollar productos que puedan ser distribuidos en el momento adecuado, con la calidad acordada, precios ajustados y con un mayor gra- do de innovación, lo cual representa una ventaja competitiva para las mismas (Evans y Linsay, 2008). Según Agulló (2000) dichas alianzas se puede clasificar de la siguiente manera:

- Alianzas comerciales: mediante esta alianza se busca lograr acuerdos en cuanto a la comercialización y distribución del producto, buscando aprovechar las economías de escala que se puedan obtener integrando las redes de distribución de las entidades, así como también buscando llegar a un mayor número de clientes, abarcando el mercado específico de cada uno de los integrantes de la alianza.

En las empresas estudiadas este tipo de alianza se da principalmente entre las plantas de alimentos y el resto de los eslabones de esta fase. Las mismas se encargan de distribuir los diferentes tipos de alimentos entre las granjas propias y alquiladas de las empresas y en algunos casos, como el de la empresa Protinal, también abastece el mercado externo.

- Alianzas tecnológicas: se refiere al intercambio de la tecnología (con la 
transferencia de conocimiento vinculada a la misma), métodos, innovación y desarrollo logrado por las entidades, buscando llevar a cabo las mejores prácticas.

Este tipo de alianza se da entre las diferentes clases de granjas que poseen esta fase. Los encargados de las mismas intercambian sus métodos y concimientos referentes a: alimentación, vacunación, limpieza de galpones, entre otras operaciones que se realizan en ellas. De igual forma, reportan ventajas y desventajas de emplear los nuevos sistemas avícolas, como son comederos y bebederos automáticos y galpones de ambiente controlado.

- Alianzas de producción: buscan el mejoramiento de los procesos, bien sea mediante el trabajo conjunto en cuanto a la creación de los productos, o a través de integraciones entre las organizaciones, con la finalidad de lograr entregas oportunas, mejorar calidad y reducir costos.

Esta es el tipo de alianza de mayor relevancia que se crea entre los eslabones de dichas empresas. Cada eslabón crea su plan de producción según las necesidades de su cliente; para conocer las mismas es necesario una constante comunicación así como la transferencia de los planes de producción mencionados. Es por medio de este trabajo en conjunto que se mantiene el ritmo de producción en esta fase de la cadena de suministro.

Sin embargo, el hecho de aplicar alianzas que mantienen integrados los eslabones de esta fase en cada empresa no garantiza que la misma sea efectiva. Para ello es necesario asegurar buenas relaciones con proveedores externos de la empresa, a través de los cuales se adquieren los materiales.
Por tal motivo, es importante considerar el tipo de estrategia de adquisición de materiales aplicada en la empresa. Dichas estrategias deben dirigirse al establecimiento de buenas relaciones proveedor-cliente a largo plazo. Bowersow et al. (2007:84) opinan que "una estrategia de adquisición eficaz para apoyar las operaciones de la cadena de suministro requiere una relación de trabajo mucho más estrecha entre los compradores y los vendedores que como debería ser en la práctica profesional". Los mismos autores reflejan que "han surgido tres estrategias: la consolidación del volumen, la integración operativa del proveedor y la administración del valor":

- Consolidación del volumen: la aplicación de esta estrategia mediante la reducción en el número de proveedores ha mejorado la eficacia en el proceso de aprovisionamiento. Al considerar esta estrategia, la adquisición se posicionó para aprovechar los negocios con los proveedores aumentando la fuerza de negociación del comprador en la relación con los mismos.

El empleo de esta estrategia trae diversos beneficios; sin embargo, el más evidente es concretar volúmenes de compras más grandes con un proveedor, permitiéndole aprovechar las economías de escala al dividir el costo fijo entre un volumen de producción más grande. Sin embargo, al usar pocas fuentes de suministro aumenta el riesgo de incidir en déficit de materiales.

Este tipo de estrategia es aplicada por las 4 empresas en estudio, con los proveedores de pollitos para los eslabones iniciales de su cadena; todas realizan pedidos de 30.000 pollitos en promedio; 
es decir, realizan pedidos de gran volumen que disminuyen el costo del mismo.

- Integración operativa del proveedor: el momento en el cual los compradores y los vendedores comienzan a integrar sus procesos y actividades en un intento del mejoramiento sustancial del desempeño, es la siguiente etapa del desarrollo de las estrategias.

Esta integración puede adoptar distintas formas, tales como: el comprador puede permitirle el acceso al proveedor a la información de ventas o de pedidos, para que el mismo esté en constante conocimiento de cuáles productos se venden, permitiéndole posicionarse para cumplir de manera eficaz los requerimientos del comprador a un costo reducido; el comprador y el vendedor pueden trabajar juntos para identificar los procesos relacionados con el suministro, buscando la manera de rediseñarlos; lo que puede mejorar la comunicación entre ambos así como reducir el tiempo del pedido. El objetivo principal de esta estrategia es eliminar el desperdicio, reducir el costo y desarrollar una relación que permita a ambas partes involucradas mejorar mutuamente sus procesos.

Esta estrategia es empleada por las empresas en estudio con los proveedores de pollitos y con los de materia prima para los alimentos. A ambos se les transmite información referente al programa de producción para que se preparen con anticipación y puedan cubrir los requerimientos de la empresa. También se les transmite información sobre el rendimiento o la calidad de los materiales que se están adquiriendo; por ejemplo, a los proveedores de pollitos se les informa so- bre índices de mortalidad, crecimiento, entre otros.

- Administración de valor: esta estragegia va dirigida a la participación de los proveedores desde el diseño del producto, de tal manera que se reduzca la complejidad del proceso, se mejore la calidad del producto y se disminuyan los costos en los que se incurran (Bowersox et al., 2007).

La investigación realizada revela que a pesar de que las empresas internamente realizan esfuerzos por obtener un producto de alta calidad aunado a bajos costos en su procesamiento, no lo hace a través de la participación en el proceso de diseño y concepción de los productos provenientes de sus proveedores; es decir, la estrategia de administración del valor no es aplicada.

Una vez analizado el canal de abastecimiento, las alianzas comerciales, tecnológicas y de producción aplicadas; además las estrategias de adquisición utilizadas, el diagrama de la fase de aprovisionamiento de la industria avícola se presenta en el Diagrama 2.

\section{Fase de procesamiento de la cadena de suministro avícola}

La fase de procesamiento de la cadena de suministro incluye todas las empresas que se encargan de transformar la materia prima en diferentes productos para los clientes. Para Casanovas y Cuatrecasas (2003:18), el procesamiento comprende la "gestión de las operaciones de fabricación de las diferentes plantas." Urzelai (2006) agrega que esta fase se encarga de procesar los productos en 
Fases de la cadena de suministro de las empresas avícolas

Aponte F., Bertila J.; González, Abraham J. y González P., Angel D.

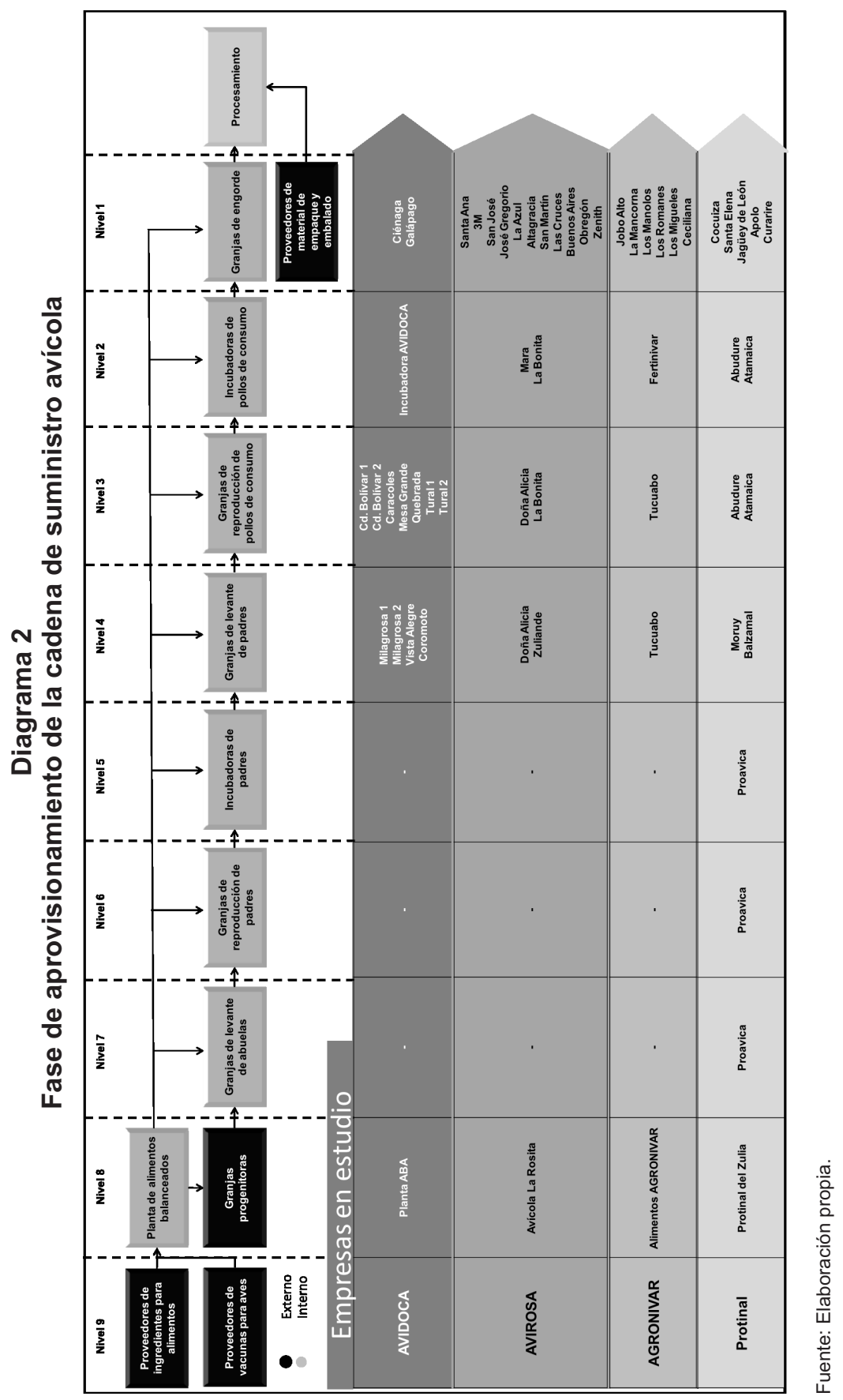


grandes lotes, en aras de evitar los cambios de producción, aprovechar las economías de escala y minimizar el costo unitario del producto.

Por su parte, Bowersox (2007:89) indica que "los fabricantes agregan valor al convertir la materia prima en productos para los clientes o la industria. Ellos crean valor al producir grupos de productos y servicios para clientes o integrantes intermedios de la cadena de suministro".

En el caso de la fase de procesamiento de la industria del sector avícola, ésta incluye las plantas que se encargan de beneficiar a las aves para obtener el pollo entero, incluyendo el proceso de evisceración por medio del cual se obtienen diferentes subproductos, tales como: corazón, hígado, cabezas, patas, entre otros. Algunas plantas tienen incorporados en sus procesos de beneficio el proceso de despresado; por medio de éste se obtienen por separado diferentes par- tes del ave, tales como: alas, pechuga, muslo, entre otros.

De igual forma, algunas plantas se encargan de procesar la carne de pollo para convertirla en productos como: carne mecánicamente deshuesada (CMD), nuggets, croquetas, entre otros conformados.

El Cuadro 3, muestra las características de las plantas que conforman esta fase. Por medio del mismo puede notarse que la empresa en estudio que tiene la mayor capacidad es Protinal del Zulia, dado que entre sus dos plantas beneficia 134.895 aves/día, seguido de AVIROSA con 66.000 aves/día. Sin embargo, la planta que tiene la mayor capacidad para producir despresados de pollo es AVIROSA con 20.800 aves/día; esto se debe a que es la única de las empresas en estudio que posee el proceso de despresado separado del proceso de beneficio; es decir, para esta empresa el proceso de despresado conforma otro eslabón de su cadena de su-

Cuadro 3

Plantas procesadoras de la industria avícola

\begin{tabular}{llcc}
\hline Empresa & Nombre & Tipo de proceso & Capacidad (aves/40sem) \\
\hline AVIDOCA & AVIDOCA & Beneficio & 50.000 \\
AVIROSA & Mara I & Despresado & 10.000 \\
& Mara II & Beneficio & 40.000 \\
& VILVA & Beneficio & 26.000 \\
& & Despresado & 20.800 \\
AGRONIVAR & AGRONIVAR & Conformado & Variable \\
& & Beneficio & 16.000 \\
PROTINAL & Empacadora & Despresado & 3.200 \\
& & beneficio & 60.030 \\
& Zupollo & Despresado & 6.670 \\
& & Beneficio & 74.865 \\
& & Despresado & 5.635 \\
\hline
\end{tabular}

Fuente: Elaboración propia. 
ministro. Además, es la única de las empresas en estudio que produce conformados de pollo (carne precocida).

Todas las plantas mencionadas en esta fase son de producción masiva o continua; es decir, trabajan bajo una estrategia de producción según planeación. Vollmann et al. (2005) indican que esta estrategia es aplicada por las empresas que explotan una economía de escala a partir de series de producción extensas. Bajo la misma se suele fabricar un gran inventario de artículos terminados en previsión de los requerimientos futuros del cliente.

Sin embargo, en algunas ocasiones especiales (festividades, ferias, entre otras) las empresas producen algunos productos bajo pedidos del cliente; tal es el caso del beneficio de gallina en diciembre o el pollo tipo parrilla. Según
Vollmann et al. (2005), esta estrategia busca producir exactamente lo especificado por los clientes, con el fin de cubrir sus solicitudes.

Una vez analizados los eslabones que forman la fase de procesamiento $y$ las estrategias con las cuales trabaja, en el Diagrama 3 se presenta el diagrama de dicha fase.

\section{Fase de distribución de la cadena de suministro avícola}

Esta fase incluye a todos los eslabones $u$ organizaciones que se encargan de conducir los productos terminados hasta el consumidor. La decisión para conducir el producto es de vital importancia; de esto depende en gran medida el grado de satisfacción dado al cliente y además garantiza

\section{Diagrama 3}

Fase de procesamiento de la cadena de suministro avícola.

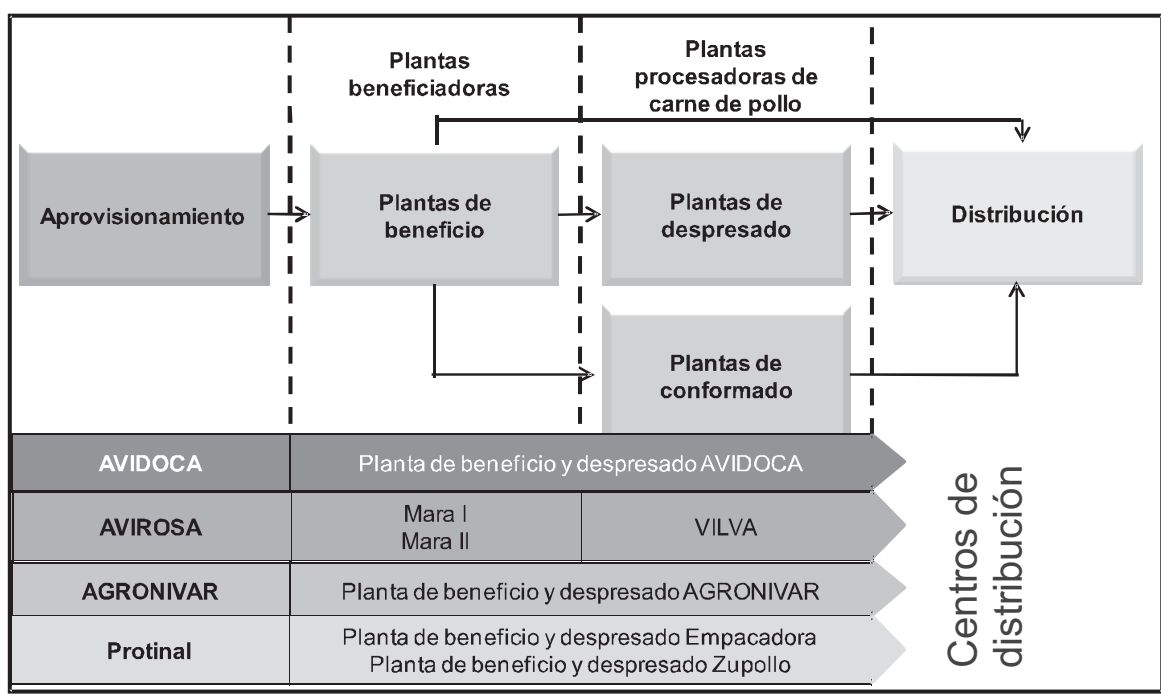

Fuente: Elaboración propia. 
que el producto esté al alcance del mismo, permitiendo la rentabilidad y perdurabilidad de la organización.

Al respecto, Serra (2005:20) opina que "en general, una de las primeras decisiones que se deben tomar es cómo llevar los productos a los consumidores. Aquí aparece el concepto de canal logístico o de suministro, que conecta al productor con el cliente". En tal sentido, Miquel et al. (2008:57) expresan que "el canal de distribución está constituido por la trayectoria que ha de seguir un bien o servicio desde su punto de origen o producción hasta su consumo, y, además, por el conjunto de personas y/o entidades que permiten la realización de las tareas correspondientes a lo largo de dicha trayectoria".

De igual manera, Mercado (2004: 320) define los canales de distribución como "los conductos que cada empresa escoge para la distribución más completa, eficiente y económica de sus productos o servicios de manera que el consumidor pueda adquirirlos con el menor esfuerzo posible".

De conformidad con lo anterior, los canales de distribución son las distintas rutas o vías integradas por personas y/o entidades a través de las cuales fluye el producto terminado, para llegar con el menor esfuerzo hasta el consumidor final, de manera que satisfaga sus necesidades, significando para la empresa menores costos y mayor eficiencia.

Antes de conocer los tipos de canales de distribución empleados en la cadena de suministro avícola, es necesario definir los elementos que lo integran:
- Productor: de acuerdo con Gitman y McDaniel (2008:492), el productor es "una organización que transforma las materias primas en productos terminados", es decir, aquella que produce el bien para lograr la satisfacción del cliente. Este elemento representa al último eslabón de la fase de procesamiento.

- Centros de distribución: según Soto et al. (2004) un centro de distribución es un lugar fijo donde se desarrollan procesos logísticos especializados de alto movimiento, en el cual las áreas de almacenamiento son reemplazadas por áreas donde se reciben, almacenan, preparan y despachan mercancías; respondiendo a las necesidades del cliente a un costo razonable. El Cuadro 4, muestra los centros de distribución que poseen las empresas en estudio. Los mismos se encargan de preparar la mercancía para distribuirla por medio de sus vendedores a sus clientes mayoristas y minoristas.

En las empresas estudiadas, los centros de distribución funcionan también como puntos de venta de productos al mayor y al detal. Es importante aclarar que la empresa AVIDOCA no posee centros de distribución ni puntos de venta. Los vendedores distribuyen sus productos desde la planta de beneficio.

- Mayoristas: de acuerdo con Quintana (2009), son las organizaciones de negocios que compran y revenden mercancías a tiendas de menudeo y otros comercios y también a usuarios industriales, institucionales y comerciales, pero no venden en cantidades importantes a los últimos consumidores. Por lo tanto, un mayorista compra el producto en grandes cantida- 


\section{Cuadro 4}

Centros de distribución de la cadena de suministro avícola

\begin{tabular}{llcc}
\hline Empresa & Nombre & Número de vendedores & Tipo de venta \\
\hline AVIDOCA & - & 14 & Al mayor \\
AVIROSA & Maracaibo I & & Al mayor y al detal \\
& Maracaibo II & & Al mayor \\
& Mara & 23 & Al mayor y al detal \\
& Punto Fijo & & Al mayor y al detal \\
& Barrio Guzmán & & Al mayor y al detal \\
& Concordia & & Al mayor y al detal \\
AGRONIVAR & AVICARNES & 9 & Al mayor y al detal \\
PROTINAL & Servifresco Maracaibo & 36 & Al detal \\
& Servifresco Ciudad Ojeda & & Al mayor y al detal \\
& Servifresco Punto Fijo & & Al mayor y al detal \\
& Servifresco El Vigia & & Al mayor y al detal \\
& Servifresco San Cristóbal & & Al mayor y al detal \\
\hline
\end{tabular}

Fuente: Elaboración propia.

des, para posteriormente revenderlo al por mayor a detallistas, otros mayoristas o a los fabricantes de otros productos.

- Minoristas: el autor anteriormente referido explica que el minorista es un comerciante, a veces agente, cuya función principal es vender directamente al consumidor último. Es decir, son aquellos que se encargan de las transacciones con el consumidor final (comercialización), vendiendo al detalle o al por menor.

- Agentes vendedores: el mismo autor define al agente como la unidad gestora de compras o ventas, que no adquiere el título de propiedad de las mercancías que trabaja, es decir, es quien va a llevar a cabo las negociaciones entre el productor y los intermediario, sin adquirir el producto.

- Consumidor: es importante realizar una distinción entre los términos cliente y consumidor. Rivera et al. (2009) explican que cliente es aquel que adquiere el producto en la empresa o tienda, pudiendo ser o no el usuario final, mientras que el consumidor es quien hace uso del producto para su beneficio personal o utilidad, por lo que las políticas de distribución van enfocadas a llamar la atención del cliente, quien será el protagonista del mensaje, aunque no deben ser perdidas de vista las preferencias del consumidor; es decir, los esfuerzos de promoción del producto deben estar dirigidos a ambos.

Una vez estudiados los componentes del canal de distribución puede presentarse su clasificación, la cual según su longitud, depende del número de intermediarios que participen. En tal sentido, Lamb et al. (2006) indican 4 posibles canales: 
- Canal directo: donde no interviene ningún intermediario. El consumidor adquiere los productos en donde es producido.

- Canal corto: intervienen los minoristas o detallistas para venderle el producto al consumidor.

- Canal largo: se presenta la intervención de los mayoristas para venderle el producto a los detallistas y éstos al consumidor final.

- Canal largo (agente-vendedor): funciona igual al anterior salvo por la intervención de los vendedores entre los mayoristas y los productores.

La investigación realizada muestra que en el caso de la industria avícola, los miembros del canal mencionados anteriormente, se combinan para formar un gran número de canales que facilitan llevar el producto al mercado. A continuación, en el Diagrama 4, se enumeran los canales empleados por las industrias en estudio para distribuir sus productos, principalmente en los estados: Zulia, Falcón, Lara, Táchira, Mérida y Trujillo.
1. Planta procesadora-consumidor: los productos son vendidos de forma directa al consumidor final.

2. Planta procesadora - centro de distribución-consumidor: el producto es llevado desde la planta hasta los diferentes puntos de venta que poseen detallistas para venderlo al consumidor final.

3. Planta procesadora - mayorista - consumidor: algunos clientes mayoristas buscan sus productos en las plantas procesadoras y poseen ventas al detal para venderlos al consumidor.

4. Planta procesadora - minorista- consumidor: el minorista busca el producto en la planta procesadora y posteriormente vende estos productos a los consumidores finales.

5. Planta procesadora - centro de distribución - mayorista - consumidor: algunos clientes mayoristas de la empresa poseen tiendas detallistas para vender al consumidor final.

6. Planta procesadora - centro de distribución - minoristas - consumidor: el

\section{Diagrama 4}

Canales de distribución de la industria avícola

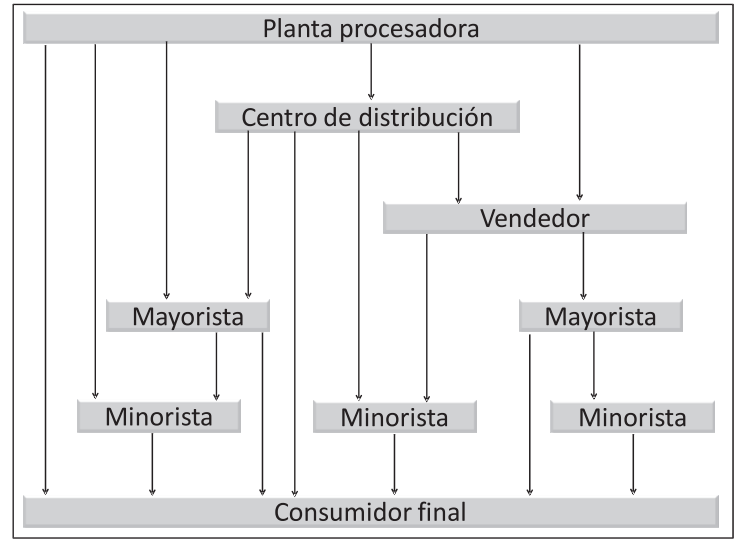

Fuente: Elaboración propia. 
producto se traslada a los distribuidores de la empresa para que sea vendido a diferentes supermercados, abastos y tiendas al menudeo, donde el consumidor pueda adquirirlos.

7. Planta procesadora - vendedor - mayorista - consumidor: algunas veces los vendedores de la empresa le llevan el producto a los mayoristas y estos se los venden al detal al consumidor final.

8. Planta procesadora - vendedor - minorista - consumidor: en algunos casos la empresa emplea sus vendedores para hacer contacto con minoristas y distribuirle el producto para que ellos lo vendan al consumidor final.

9. Planta procesadora - mayorista - minoristas - consumidor: algunos clientes mayoristas buscan sus productos en las plantas procesadoras para luego venderlos a minoristas y ellos al consumidor final.

10. Planta procesadora - centro de distribución- mayoristas - minoristas - consumidor: este canal se produce de la combinación de los dos anteriores.

11. Planta procesadora - vendedor - mayorista - minorista - consumidor: en algunos casos la empresa emplea sus vendedores para hacer contacto con mayoristas, estos a su vez le distribuyen el producto a minoristas para que lo vendan al consumidor final.

12. Planta procesadora - centro de distribución- vendedor - mayoristas - consumidor: este canal se da cuando los vendedores sacan los productos de los puntos de venta para llevarlos a mayoristas que venden productos al detal.

13. Planta procesadora - centro de distribución - vendedor - minorista - consu- midor: algunos clientes minoristas se atienen por medio de vendedores.

14. Planta procesadora - centro de distribución- vendedor - mayorista - minorista - consumidor: este es el canal de mayor longitud, producto de la intervención de todos los intermediarios ya mencionados.

En el Cuadro 5, se pueden observar cuáles canales emplea cada empresa en estudio. Protinal del Zulia es la empresa que mayor cantidad de canales emplea para distribuir su producto en el mercado con un total de 13 , seguido de AVIROSA con 12 canales de distribución. Puede notarse que el mismo orden que tenían las empresas en cuanto a su capacidad de producción, lo tienen en cuanto a la cantidad de canales de distribución empleados; es decir, la empresa que más produce es Protinal, por ende emplea más canales para distribuir sus productos, en contraparte AGRONIVAR es la empresa que menor cantidad de productos elabora y por ello necesita de menos canales para distribuirlos en el mercado.

De igual forma se puede observar que los canales emplean una gran cantidad de intermediarios (mayoristas, minoristas, vendedores) para distribuir sus productos; es decir, emplean una distribución intensiva para cubrir el mercado. Serra (2005:23) define la distribución intensiva como la "venta a través del máximo de puntos de venta posibles". Asimismo, Lamb et al. (2006:318) afirma que ésta es "una forma de distribución dirigida a una cobertura de mercado máxima. El fabricante trata de tener el producto disponible en cada punto de venta donde clientes potenciales podrían querer comprarlo". 


\section{Canales de distribución por empresa}

\begin{tabular}{|c|c|c|c|c|c|c|}
\hline Canal & Longitud & $\begin{array}{c}\text { Cantidad de } \\
\text { intermediarios }\end{array}$ & AVIDOCA & AVIROSA & PROTINAL & AGRONIVAR \\
\hline 1 & Directo & 0 & & & & $x$ \\
\hline 2 & Corto & 1 & & $x$ & $x$ & \\
\hline 3 & & & $x$ & $x$ & $x$ & \\
\hline 4 & & & $x$ & & $x$ & \\
\hline 5 & Largos & 2 & & $x$ & $x$ & \\
\hline 6 & & & & $x$ & $x$ & \\
\hline 7 & & & $x$ & $x$ & $x$ & $x$ \\
\hline 8 & & & $x$ & $x$ & $x$ & $x$ \\
\hline 9 & & & $x$ & $x$ & $x$ & \\
\hline 10 & & 3 & & $x$ & $x$ & \\
\hline 11 & & & $x$ & $x$ & $x$ & $x$ \\
\hline 12 & & & & $x$ & $x$ & \\
\hline 13 & & & & $x$ & $x$ & \\
\hline 14 & & 4 & & $x$ & $x$ & \\
\hline & Total & 6 & 12 & 13 & 4 & \\
\hline
\end{tabular}

Fuente: Elaboración propia.

Finalmente, es importante aclarar que esta es la fase de la cadena de suministro avícola que se encuentra menos integrada. El estudio revela que sólo algunas de las empresas poseen centros de distribución propios que se encargan de distribuir los productos al mayor o de venderlo al detal. La mayoría emplea vendedores que se encargan de distribuir el producto en el mercado y una vez vendidos se pierde el control sobre los mismos.

Por lo antes mencionado, se puede afirmar que los canales de distribución de esta fase de la cadena son independientes. Según Miquel et al. (2006) dichos canales son aquellos en los que sus componentes o elementos no se encuentran re- lacionados, por lo que cada uno adopta sus políticas de venta de manera independiente, una vez que cada una de las empresas vende el producto pierde cualquier tipo de control sobre el mismo. La organización que lo adquiere, ya sea mayorista o minorista, puede vender su producto en el precio, cantidad y condiciones que quiera (recordando que el pollo es un producto regulado); incluso la mayoría de las veces pueden venderlo a quien ellos deseen.

Una vez estudiados los diferentes canales de distribución utilizados, la forma en que éstos se encuentran integrados y su intensidad de distribución, el diagrama de la fase se presenta en el Diagrama 5. 


\section{Diagrama 5}

\section{Fase de distribución de la cadena de suministro avícola}

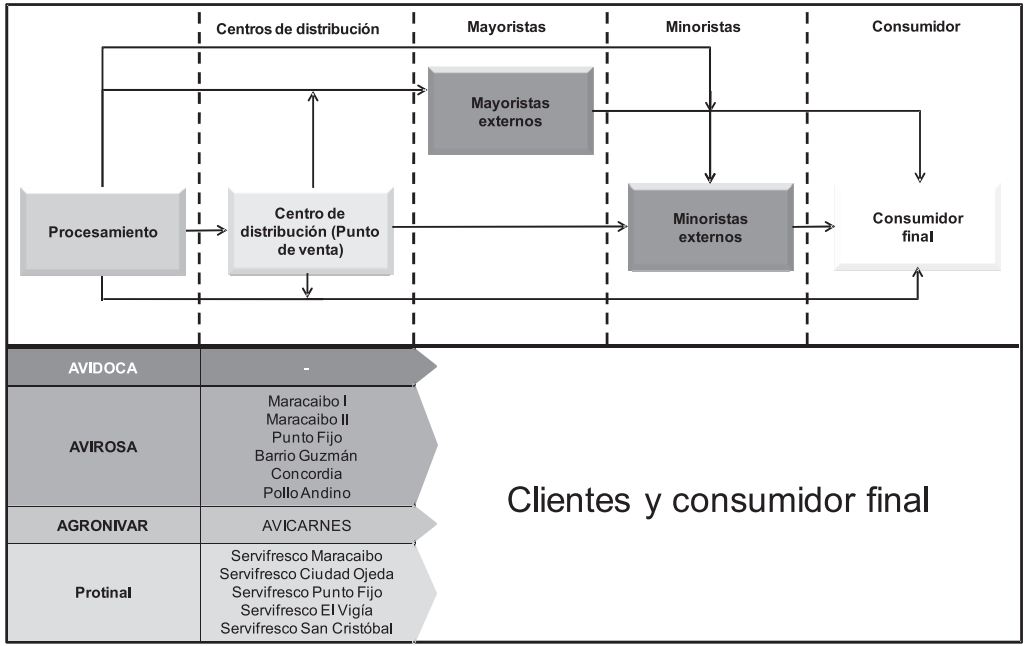

Fuente: Elaboración propia.

\section{Conclusiones}

La cadena de suministro avícola está integrada por 15 eslabones distribuidos en tres fases: aprovisionamiento, procesamiento y distribución. El sector avícola es uno de los pocos sectores en el país que posee su cadena de suministro casi en su totalidad; al tener integrado 10 de sus 15 eslabones aumenta la fluidez a sus procesos para dar respuesta a los requerimientos de los clientes en la cantidad, momento y condiciones requeridas, a un bajo costo.

La fase de aprovisionamiento contiene mayor cantidad de eslabones y se encuentra parcialmente integrada por razones principalmente económicas; es decir, algunas empresas prefieren adquirir su materia prima en el nivel 9 del canal de abastecimiento o en el 5, con la finalidad de incurrir en menos costos de producción. Sin embargo, los problemas actuales en la adquisición de divisas algunas veces dificultan la adquisición de materia prima, lo que genera ciertos retrasos en la producción.

La fase de procesamiento es el corazón productivo de la cadena de suministro. Al ser un producto de consumo masivo, es necesaria la aplicación de la estrategia de producción continua para satisfacer las necesidades del mercado de forma ininterumpida. Las empresas se encuentran en una etapa de transformación para automatizar los procesos en esta fase, de tal manera que se aumente la rata de producción y se obtengan productos de mayor calidad.

En la fase de distribución, la industria avícola emplea en total 14 canales para la distribución de sus productos. Es la fase de la cadena que se encuentra menos integrada con la finalidad de poder 
abarcar el mercado de forma intensiva. Sin embargo, las relaciones entre los centros de distribución de las empresas y los mayoristas y minoristas son muy estrechas, con la finalidad de dar respuesta a los requerimientos del cliente.

En síntesis, el alto grado de integración en la cadena de suministro avícola, aunado a las estrategias aplicadas en las fases de procesamiento y distribución, están dirigidas a satisfacer el mercado y a mantenerse competitivo en el mismo; sin embargo, las estrategias aplicadas en la fase de aprovisionamiento se ven afectadas por las políticas cambiarias del país, afectando así el nivel de productividad de toda la cadena. Éste es uno de los principales factores que generan la escasez de los productos avícolas en el mercado.

\section{Referencias Bibliográficas}

Agulló, Fernando (2000). "Las alianzas estratégicas, una respuesta a la demanda global". Economía Industrial (Revista en línea). $\mathrm{N}^{\circ}$ 331. pp. 11-15. Disponible en http://www.mityc.es [Consulta: 2011, Diciembre 05].

Ballou, Ronald (2004). Logística: administración de la cadena de suministro. Quinta edición. México. Pearson Educación.

Bowersox, Donald; Closs, David y Cooper, Bixby (2007). Administración y logística en la cadena de suministro. Segunda edición. México D.F. Mc Graw-Hill.

Casanovas, August y Cuatrecasas, Luis (2003). Logística empresarial. Primera edición. Barcelona, España. Gestión 2000.

Chirinos, Alira; Rodríguez, Guillermo y Bonomie, María (2008). "Integración verti- cal de la cadena de valor del sector avícola en la empresas del estado Zulia". Revista Venezolana de Análisis de Coyuntura. Volumen XIV, número 001, pp. 175-193.

Chopra, Sunil y Meindl, Peter (2008). Administración de la cadena de suministro. Estrategia, Planeación y Operación. Tercera edición. México. Pearson Educación.

Christopher, Martin (2008). Logística: aspectos estratégicos. Segunda Edición. Limusa. México.

Evans, James y Lindsay, William (2008). Administración y Control de la Calidad. Séptima edición. México. Cengage learning.

Gitman, Lawrence y McDaniel, Carl (2008). EI futuro de los negocios. Quinta Edición. México. Cengage Learning.

Hernández, Roberto; Fernández, Carlos y Baptista, Pilar (2006). Metodología de la Investigación. Cuarta edición. México D.F. McGraw-Hill.

Hurtado, Jacqueline (2007). El proyecto de investigación: Metodología de la Investigación Holística. Quinta edición. Caracas, Venezuela. Quirón Ediciones.

Instituto Nacional de Estadística INE (2011). Informe del sector avícola venezolano. Disponible en http://www.ine.gov. ve. [Consulta: 2011, Octubre 10].

Lamb, Charles; Hair, Joseph y McDaniel, Carl (2006). Fundamentos de marketing. Cuarta edición. España. Editorial Thomson.

Miquel, Salvador; Parra, Francisca; Lhemie, Christian y Miquel, María (2008). Distribución comercial. Sexta edición. México. Pearson Educación.

Mercado, Salvador (2004). Mercadotecnia programada. Segunda edición. México. Editorial Limusa. 
Pires, Silvia y Carretero, Luis (2007). Gestión de la cadena de suministro. Primera edición. España. McGraw-Hill.

Quintana, Miguel (2009). Fundamentos del Marketing [Artículo en línea]. Disponible en: http://cvb.ehu.es/open course_ware. [Consulta: 2011, Noviembre 09].

Rivera, Jaime; Arellano, Rolando y Molero, Víctor (2009). Conducta del consumidor. Segunda edición. España. Esic Editorial.

Serra, Daniel (2005). La logística empresarial en el nuevo milenio. España. Gestión 2000.
Soto, Astrid; Castaño, Catalina y Vásquez, Andrés (2004). Propuesta metodológica y ejemplo de aplicación para la localización y dimensionamiento de un centro de distribución. Medellín, Colombia. McGraw-Hill.

Urzelai, Aitor (2006). Manual básico de logística integral. España. Ediciones Días de Santos.

Vollmann, Thomas; Berry, Williams; Whybark, Clay y Jacobs, Robert (2005). Planeación y control de la Producción: Administración de la cadena de suministro. Quinta edición. México. McGraw-Hill. 